\title{
Revisiting pulmonary diseases using microfluidic technology
}

\begin{abstract}
Pulmonary diseases such as chronic obstructive pulmonary disease, asthma and tuberculosis are among the leading causes of death globally, accounting for nearly 4 million of all deaths worldwide every year. Prevention, management and treatment of these deadly diseases require early detection of pathogens and accurate study of pathophysiology of the respiratory system. In recent years, microfluidic technology has emerged as a powerful tool for recapitulation of the physiological and pathological scenarios of the lung for enhancing the current understanding of pulmonary disorders. This review paper outlines the advantages of microfluidic devices in providing improved insights into the mechanism of the pulmonary diseases, identification of their novel biomarkers and the development of new and advanced therapeutic strategies. Furthermore, several microfluidic lung models are discussed along with progresses made from the aboriginal to till date.
\end{abstract}

Volume I Issue I - 2016

\section{Anjali, Suruchi Poddar and Sanjeev Kumar Mahto \\ Tissue Engineering and Biomicrofluidics Laboratory, Indian Institute of Technology, India}

Correspondence: Sanjeev Kumar Mahto, Tissue Engineering and Biomicrofluidics Laboratory, School of Biomedical Engineering, Indian Institute of Technology (Banaras Hindu University), Varanasi - 221005, Uttar Pradesh, India, Tel +917617052884, Email skmahto.bme@iitbhu.ac.in

Received: August 09, 2016 | Published: September 30, 2016

Keywords: pulmonary diseases, pathophysiology, microfluidic devices, biomarkers, therapeutic strategies

Abbreviations: POCD, point-of-care device; COPD, chronic obstructive pulmonary disease; ARDS, acute respiratory distress syndrome; WHO, world health organization; TB, tuberculosis; BlaC, $\beta$ lactamase; CF: Cystic Fibrosis; CFTR: Cystic Fibrosis Trans membrane conductance Regulator; DNA: deoxyribonucleic acid; Mtb, mycobacterium tuberculosis; 3D, three-dimensional; PDMS, polydimethylsiloxane; PCR, polymerase chain reaction

\section{Introduction}

The human respiratory system consists of nose, pharynx (throat), larynx (voice box), trachea (windpipe), bronchi, and the lungs. ${ }^{1}$ The nose, pharynx, larynx, trachea and bronchi together form the respiratory airways. The bronchus continues to divide within lung into many narrower and shorter airways known as bronchioles. ${ }^{2}$ The branching networks of pulmonary airways are lined with a viscous liquid film secreted by airway epithelial cells. ${ }^{3}$ Terminal bronchioles are clustered in grapelike sacs, the alveoli. Gaseous exchange between air and blood takes place in the alveoli. The lungs contain approximately 500 million alveoli, each about 200 to $300 \mu \mathrm{m}$ in diameter. The alveolar wall consists of a single layer of type I alveolar cells. Each alveolus is surrounded by a network of pulmonary capillaries. The interstitial space between pulmonary capillaries and alveolus forms a very thin barrier which facilitates gaseous exchange. Apart from type I alveolar cells, $5 \%$ of the alveolar surface epithelium is covered by type II alveolar cells, which secrete a phosphor lipoprotein complex known as pulmonary surfactant that facilitates lung expansion. ${ }^{2}$ In addition to type II alveolar cells, surfactant proteins are also produced by Clara cells of peripheral airways. The pulmonary surfactant produced from these cells is absorbed into the luminal air-liquid interface of the pulmonary airways and thus reduces the surface tension to values as low as zero during expiration. This in turn prevents the airway closure. ${ }^{4}$ Lung epithelium is a highly complex tissue where epithelial cells, sub epithelial fibroblasts and the extracellular matrix of the airway wall are intricately involved in maintaining and regulating the structure and function of the lung. ${ }^{5}$

\section{Lung diseases}

Respiratory diseases and disorders encompass pathological conditions that mostly affect the organs and tissues that take part in gaseous exchange (Figure 1). Notably, such abnormalities affect the airways, the physiology structure of lung tissue, and the pulmonary circulation. ${ }^{6}$ The lung diseases are broadly classified into obstructive, restrictive and infectious diseases (Table 1).

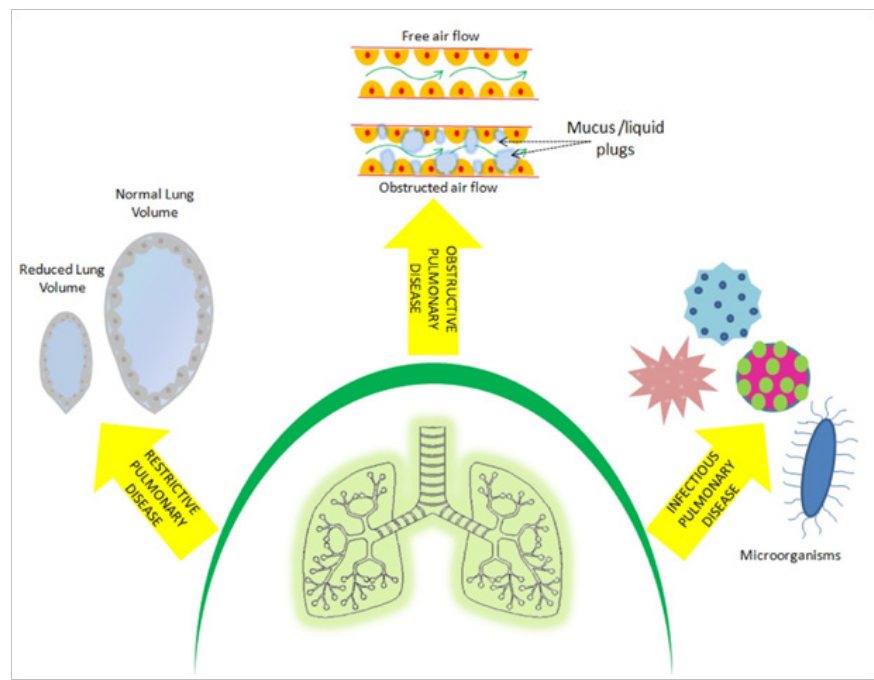

Figure I Schematic represents the focus areas that are discussed in this review paper. Broadly, we attempt here to address the versatility of microfluidic technology in creating the near-human in vitro models of pulmonary diseases including restrictive, obstructive and infectious categories.

Chronic obstructive pulmonary disease (COPD) is the fourth leading cause of death in the world. The COPD burden is projected to increase globally in the coming decades because of continued exposure to COPD risk factors and aging of the population. ${ }^{7}$ COPD, a chronic inflammatory disease, is generally an adult age problem ${ }^{8}$ and 
characterized by breathing difficulty as a consequence of narrowed airways. Long term exposure of lungs to noxious particles or gases, such as cigarette smoke, is considered as the main cause of COPD.

Table I Classification of the respiratory diseases and their conventional diagnostic methods
According to WHO, COPD will become third leading cause of death by $2030 .{ }^{9}$

\begin{tabular}{ll}
\hline Type of diseases & Respiratory diseases \\
\hline & Chronic obstructive pulmonary disorder (COPD) \\
Obstructive diseases & Asthma \\
& Acute respiratory distress syndrome (ARDS) \\
Restrictive diseases & Cystic fibrosis \\
Infectious diseases & Tuberculosis \\
\hline
\end{tabular}

Similarly, asthma is also amongst the chronic inflammatory disorders characterized by active hyper responsiveness to a variety of stimuli e.g. airborne allergens. It results from a complex interaction among inflammatory cells, mediators and airways. ${ }^{8}$ According to the most recent revised global estimate of asthma, it is found that as many as 334 million people have asthma, and that the burden of disability is high. ${ }^{10}$

In acute respiratory distress syndrome (ARDS), the integrity of the alveolar-capillary barrier is compromised due to fatal events such as severe chest trauma, serious infection in the blood or other tissues, and bacterial or viral insult to the lung. Consequently, a proteinaceous fluid leaks from capillaries into the interstitium and floods alveoli. Furthermore, inflammatory cells, neutrophils, and macrophages infiltrate alveoli and airways. The presence of fluid and inflammatory mediators alters concentration and composition of the lung surfactant and accounts for surfactant malfunction in ARDS. ${ }^{11}$

Tuberculosis (TB) is an infectious disease, caused by Mycobacterium tuberculosis. It is one of the most deadly diseases and that kills over one million people each year and infects one-third of the world's population. ${ }^{12}$ The WHO's goal to eradicate tuberculosis by 2050 is not still attainable due to limitations of current technologies for diagnosis, treatment and prevention. The WHO had estimated to have more than 2 million new cases of tuberculosis between 2011 and 2015. ${ }^{13}$

\section{Conventional diagnostic methods}

Spirometry, Cell migration assays including trans-well assay and the under-agarose assay

Patient's medical history, spirometry test, physical examinations

Gene expression analysis.

Flow-cell based system.

Acid-fast smear using sputum, interferon gamma release assay.

Cystic fibrosis (CF) patients suffer from a defect in the cystic fibrosis trans membrane conductance regulator (CFTR) gene. As a consequence, the mucus layer in the conductive airways becomes very viscous and the mucociliary clearance mechanisms are highly impaired. This results in frequent and recurrent infections of the $\mathrm{CF}$ airways, with risk of pneumonia. ${ }^{14}$

\section{Conventional study models and diagnostic methods}

Timely and effective management and control of many pulmonary diseases remain suffered from the lack of proper preventive care and treatment till date. Despite intensive research efforts, the mechanism of the pulmonary diseases remains poorly understood, mainly due to lack of suitable in vitro models that recapitulate the complex in vivo conditions accurately.

Conventional assay methods used for diagnosis of the pulmonary disease primarily include spirometry, cell migration assay, ${ }^{9}$ acid fast smear using sputum, ${ }^{12}$ gene expression analysis, ${ }^{15}$ cell culture assays, ${ }^{16-17}$ interferon gamma release assay. ${ }^{18}$ In addition, for understanding the mechanism of pulmonary diseases, researchers have conducted their studies conventionally in human patients, ${ }^{9}$ animal models, ${ }^{4,14}$ and using in vitro lung tissue slices. ${ }^{16}$ The specific limitations of the conventional study models as well as of the conventional diagnostic methods are summarized in (Table 2) (Table $3)$, respectively.

Table 2 Limitations of the conventional study models used for diagnosis of the lung diseases

\begin{tabular}{ll}
\hline In Vitro study models & Limitations \\
\hline $\begin{array}{l}\text { Animal models and human } \\
\text { patients }\end{array}$ & $\begin{array}{l}\text { Small airways in orders of millimetres cannot be adequately studied in animal models and human } \\
\text { patients, }{ }^{9} \text { expensive and raises ethical concerns. Animal models do not show immunological responses similar } \\
\text { to the level of human. }{ }^{14}\end{array}$ \\
In-Vitro lung tissue slice culture & Human organ slices can be difficult to obtain and the survival of slices is limited to a few days. ${ }^{16}$ \\
\hline
\end{tabular}

Table 3 Conventional diagnostic methods and their shortcomings

\begin{tabular}{ll}
\hline Conventional diagnostic methods & Shortcomings \\
\hline Spirometry & Requires patient cooperation that can be difficult for children or patients with severe condition. \\
Cell migration assay & Unable to control gradient. \\
Acid fast smear using sputum & Takes few months to become positive with sensitivity of $20-80 \% .{ }^{12}$ \\
Gene expression analysis & Laborious, samples are subjected to considerable ex-vivo perturbation resulting in documented phenotypic \\
Cell culture assay & and functional changes. ${ }^{15}$ \\
Interferon gamma release assay & Unable to mimic the in vivo conditions, ${ }^{16,17}$ contamination is a big challenge. ${ }^{17}$ \\
\hline
\end{tabular}




\section{Opportunities for microfluidic technologies to explore pulmonary diseases}

Developing a physiologically and anatomically accurate mode of the pulmonary system mimicking both the cellular structure and physiological function of the lung is among the daunting challenges of modern respiratory pathophysiology. Over the past few decades, several research groups have made sincere efforts to design and develop biomimetic microfluidic systems closer to the physiological scenarios of the lung structure and their function for highlighting the molecular processes underlying pathophysiology of the respiratory diseases (Table 4) ${ }^{6}$ Microfluidic systems have been used widely as platforms for the culture of mammalian cells to study various physiologic and pathologic conditions in vitro. The ability of microfluidics to recapitulate the in-vivo condition has made it popular among researchers. ${ }^{11}$ This advanced technology provides excellent platforms for identifying novels biomarkers, developing new insight into mechanisms of the disease, testing new drug candidates. Moreover, lung inflammatory diseases can be studied over several weeks in such micro-devices incorporated with cells harvested from both normal and diseased persons. ${ }^{9}$

Table 4 Summary of opportunities for microfluidic technology to explore pulmonary diseases

\begin{tabular}{|c|c|c|c|}
\hline $\begin{array}{l}\text { Type of } \\
\text { diseases }\end{array}$ & Respiratory diseases & Scenario mimicked & Technique employed \\
\hline \multirow{4}{*}{$\begin{array}{l}\text { Obstructive } \\
\text { diseases }\end{array}$} & $\begin{array}{l}\text { Chronic obstructive } \\
\text { pulmonary disorder (COPD) }\end{array}$ & $\begin{array}{l}\text { Neutrophil infiltration into airways } \\
\text { through chemo tactic migration }\end{array}$ & $\begin{array}{l}\text { The pressure difference between the inlet and outlet } \\
\text { wells establish gradient flow of the chemo attractant }{ }^{26}\end{array}$ \\
\hline & Asthma & $\begin{array}{l}\text { Detection of asthma based on } \\
\text { cellular function }\end{array}$ & Detection of asthma based on cellular function \\
\hline & $\begin{array}{l}\text { Acute respiratory distress } \\
\text { syndrome (ARDS) }\end{array}$ & Liquid plug formation & $\begin{array}{l}\text { Air tank, airflow meter, valves, syringe pumps, and a } \\
\text { computer is integrated to the microfluidic platform }{ }^{21}\end{array}$ \\
\hline & & $\begin{array}{l}\text { Repeated opening and closure of } \\
\text { airways }\end{array}$ & $\begin{array}{l}\text { Automated fraction controller integrated with the } \\
\text { microfluidic device }\end{array}$ \\
\hline \multirow{2}{*}{$\begin{array}{l}\text { Restrictive } \\
\text { diseases }\end{array}$} & \multirow{2}{*}{ Cystic fibrosis } & Mucus plug in CF bronchi & $\begin{array}{l}\text { Cell culture chamber separated by a membrane, and a } \\
\text { thick mucus layer simulated by using alginate hydroge }\left.\right|^{23}\end{array}$ \\
\hline & & Oxygen tension in $\mathrm{CF}$ airways & $\begin{array}{l}\text { Compartmentalized platform shows connections } \\
\text { between oxygen rich and oxygen depleted regions }{ }^{14}\end{array}$ \\
\hline \multirow{2}{*}{$\begin{array}{l}\text { Infectious } \\
\text { diseases }\end{array}$} & \multirow{2}{*}{ Tuberculosis } & Development of a diagnostic system & $\begin{array}{l}\text { Paper based microfluidic using salt-induced gold } \\
\text { nanoparticle (AuNP) }{ }^{25}\end{array}$ \\
\hline & & $\begin{array}{l}\text { Detection of a reporter enzyme } \\
\mathrm{BlaC}\end{array}$ & $\begin{array}{l}\text { A circular microfluidic device for capturing cytokine } \\
\text { secreting CD4 cells using glutaraldehyde }{ }^{12}\end{array}$ \\
\hline
\end{tabular}

A variety of pulmonary diseases such as COPD, asthma, ARDS are profoundly associated with the surfactant dysfunction that leads to liquid plug formation across the airway lumen. ${ }^{19}$ Several animal model studies have shown during such lung disorders severe tissuelevel damage to the distal lung airways due to repeated closure and reopening process. ${ }^{4}$ To mimic exactly the in vivo conditions, Huh et al. ${ }^{20}$ developed a compartmentalized microfluidic airway models and demonstrated that the reopening of occluded microfluidic airway causes severe injury of pulmonary epithelial cells. ${ }^{20}$ In the lung airways, rupturing of the liquid plugs leads to abnormal breath sounds known as crackles. To simulate this scenario, a three-dimensional (3D) microfluidic device was developed to detect acoustically the crackling sound and it was demonstrated that there is a higher risk of cell injury when liquid plugs become very thin. They demonstrated cellular level of lung injury under flow condition using this device. ${ }^{19}$ To create on-chip liquid plugs, various components including an air tank, airflow meter, valves, syringe pumps, and a computer are integrated to the microfluidic platform. ${ }^{21}$ Surfactant is known to reduce the surface tension during expiration to stabilize the liquid present in the airway. To investigate the physiological role of surfactant, a microfluidic model of small airway of the peripheral lung was recently developed and it was shown that addition of a physiological concentration of surfactant to the propagating liquid plug protects epithelium and reduces cell death significantly. ${ }^{4}$ Despite much intensive research using the conventional models, the responses of airway barrier to environmental agents remained poorly understood.
Towards understanding such airway behaviors Blume et al. ${ }^{22}$ recently developed a novel dynamic 3D in vitro airway epithelium model in which they integrated the microfluidic culture system with automated fraction collector that allows more sensitive early phase analysis of barrier responses to environmental impacts. ${ }^{22}$

Similarly, more advanced platforms of models airways specific to cystic fibrosis (CF) have recently surfaced. Traditionally, flow-cell based system has been used for studying the mechanism of cystic fibrosis. However, such system lacks the in-vivo environment of the lung airways. Skolimowski $\mathrm{M}$ et al. ${ }^{23}$ developed a microfluidic model of CF bronchi. The model consists of 2 cell culture chambers separated by a membrane. The thick mucus layer spotted in the CF patients was simulated by an alginate hydrogel above the membrane and the epithelial cells were cultured in the bottom chamber. They inoculated Pseudomonas aeruginosa PAO1 strain to the hydrogel layer for studying the antibiotic treatment on bacterial infection. ${ }^{23}$ Also, the same groups designed a modular microfluidic airway model that simulates the oxygen tension in different compartments of the $\mathrm{CF}$ airway. This model permits to freely reconfigure connections between oxygen rich and oxygen depleted regions. It was able to mimic different scenarios such as clogging of the ostia in recurrent sinusitis or the development of mucus plug in the bronchioles that was previously not possible using a flow-cell based system. ${ }^{14}$

For an effective and efficient pathogen detection method, assays should ideally be cost-effective, fast, sensitive, and accurate. A 
microfluidic device has the ability to improve the performance of assays by facilitating less consumption of reagents, rapid analysis, high reliability and sensitivity as well as integrating multiple processes in a single device. ${ }^{24}$ Therefore, microfluidic technology has also been exploited to design and develop various types of micro-devices for the diagnosis of tuberculosis (TB), pneumonia and asthma.

Treatment of TB essentially requires effective detection of infectious pathogen.$^{24}$ Tsai et al. ${ }^{25}$ presented a microfluidic paper based diagnostic device that utilized salt-induced gold nanoparticle (AuNP) based colorimetric diagnosis of TB DNA sequences. This device has shown the potential to facilitate high-throughput and high-content screening and help avoid the requirement of sophisticated optical based detection equipment as well as a well-trained technician. ${ }^{25} \mathrm{At}$ present, the biggest challenge with the treatment of TB is its prompt diagnosis. WHO estimated tuberculosis detection rate to be just $63 \% .^{12}$ For this purpose, Rosenfeld et al. ${ }^{12}$ developed a novel microfluidic device for rapid, sensitive, specific and quantitative detection of a reporter enzyme, BlaC specifically produced from Mycobacterium tuberculosis (Mtb). ${ }^{12}$ Similarly, a circular microfluidic device was designed and presented for the accurate diagnosis of Mtb by capturing the cytokine secreting CD4 cells using glutaraldehyde. ${ }^{18}$

Asthma is very difficult to accurately diagnose because its symptoms can be transient and common tests are susceptible to user error. For accurate diagnosis of asthma, a microfluidic device was recently developed that provides an alternative platform for the clinicians to characterize asthma based on the cellular function. They showed that neutrophils move slower than normal in response to inflammation among asthmatics compared to those with allergic rhinitis. ${ }^{26}$ Also, COPD correlates well to neutrophil infiltration into airways through chemo tactic migration. Based on the same physiological phenomena, a microfluidic platform was developed for evaluating neutrophil chemotaxis to sputum samples taken from a COPD patient. This assay furthermore revealed an increased level of neutrophil chemotaxis as observed physiologically in COPD patients. ${ }^{9}$

\section{Discussion}

The cone-shaped, sponge-like organs, lungs, represent an extraordinary example of natural air filter for facilitating oxygen supply to the human body. To redesign or reproduce them either partly or to the full capacity, achieving the physiological-level architecture and complexities is an important issue and therefore considered one of the most challenging tasks. Several research groups have dared to confront the situation with the help of microfluidic technology. Undoubtedly, some of the best innovations including a small (merely few centimeters in size) flexible chip mimicking the alveolar level intricacies have come up in the past few years. Such devices are aimed to replenish the need of those who suffer from compromised breathing because of severe pulmonary diseases. This technology thus facilitates gaining an insight into the pathophysiology of the lung complications and in turn possibly help improve the therapeutic approaches while dealing with potentially fatal pulmonary diseases.

Although microfluidics is explored as an alternative platform for culturing cells in vitro, it has its own limitations that one cannot overlook while performing cell culture studies. Considering its advantages of design flexibility, lesser amount of laboratory reagents consumption, real-time analysis in spatial and temporal resolution, the typical challenges associated with these devices cannot be ignored. A major hurdle that accompanies every microfluidic device is the hydrophobicity of its polymeric material e.g., polydimethylsiloxane
(PDMS). Due to its inherent hydrophobic nature, surface treatment of PDMS devices becomes an important and necessary step to mimic the hydrophilic physiological environment for mammalian cell culture. This can be overcome by exposing the culture platform with plasma rays that render the surface of PDMS hydrophilic. ${ }^{27}$ Secondly, culture protocols need to be optimized at every step for microfluidic-specific conditions; as long as minute volumes of reagents are involved as it needs patience and careful handling. Creating a dynamic system inside the microfluidic device ask for some additional components such as syringe pumps and its accessories that require expertise and dedication to gain full operational control. ${ }^{28}$ Recreation of artificial structures with physiological-level resemblance demands microarchitectured chips. Designing of these complex microarchitectures can get better with some professional softwares specifically dedicated for this purpose. In spite of being stymied by such barriers, microfluidics still stands as one of the most exploited technologies for conducting experiments that imitates physiological-level and disease-specific microenvironments.

\section{Conclusion}

The microfluidic technology has been exploited to create numerous excellent platforms that recapitulate the in vivo conditions of the lung environment very closely and therefore facilitate better insight of the physiological and pathological conditions of the lungs. Such innovations ultimately have enhanced the current understanding of pulmonary disorders and thus may help establishing improved guidelines for developing therapeutic interventions. Nevertheless, there remains a critical need to design and develop disease-specific point-of-care devices (POCDs) using microfluidic technology for the early, effective and onsite diagnosis of pulmonary diseases.

\section{Acknowledgements}

This work was financially supported by a DST-INSPIRE (DST/ INSPIRE/04/2013/000836) research grant from the Department of Science and Technology, Government of India.

\section{Conflict of interest}

The author declares no conflict of interest.

\section{References}

1. GJ Tortora, B Derrickson. Principles of anatomy and physiology. 12th ed. NJ, USA: Wiley, Hoboken; 2009

2. S. Price, L Sherwood. Study guide: human physiology: from cells to systems. 7th ed. Brooks/Cole, Belmont, California, USA: Cengage Learning; 2010.

3. WC Tian, E Finehout. Microfluidics for biological applications. In: Lecture notes in control and information sciences vols. (Illustrated), LLC, New York, USA: Springer Science \& Business Media; 2009.

4. Tavana H, Zamankhan P, Christensen PJ, et al. Epithelium damage and protection during reopening of occluded airways in a physiologic microfluidic pulmonary airway model. Biomed Microdevices. 2011;13(4):731-742.

5. Punde TH, Wu WH, Lien PC, et al. A biologically inspired lung-on-achip device for the study of protein-induced lung inflammation. Integr Biol (Camb). 2015;7(2):162-169.

6. Perestrelo R, Águas ACP, Rainer A, et al. Microfluidic organ/body-ona-chip devices at the convergence of biology and microengineering. Sensors (Basel). 2015;15(12):31142-31170. 
7. Vestbo J, Hurd SS, Agustí AG, et al. Global strategy for the diagnosis, management, and prevention of chronic obstructive pulmonary disease: GOLD executive summary. Am J Respir Crit Care Med. 2013;187(4):347-365.

8. Jindal SK, Aggarwal AN, Gupta D, et al. Indian study on epidemiology of asthma, respiratory symptoms and chronic bronchitis in adults (INSEARCH). Int J Tuberc Lung Dis. 2012;16(9):1270-1277.

9. Wu J, Hillier C, Komenda P, et al. A microfluidic platform for evaluating neutrophil chemotaxis induced by sputum from COPD patients. PLoS ONE. 2015;10(5):e0126523.

10. The Global Asthma Report; 2014.

11. Tavana H, Huh D, Grotberg JB, et al. Microfluidics, lung surfactant, and respiratory disorders. LabMedcine. 2009;40(4):203-209.

12. Rosenfeld L, Cheng Y, Rao J, et al. Rapid detection of tuberculosis using droplet-based microfluidics. Microfluidics, Bio MEMS, and Medica Microsystems XII, San Francisco, California, USA: Springer; 2014.

13. Wang $\mathrm{S}$, Inci $\mathrm{F}$, De Libero $\mathrm{G}$, et al. Point-of-care assays for tuberculosis: role of nanotechnology/microfluidics. Biotechnol Adv. 2013;31(4):438449.

14. Skolimowski M, Weiss Nielsen M, Abeille F, et al. Modular microfluidic system as a model of cystic fibrosis airways. Biomicro fluidics. 2012;6(3):34109.

15. Warner EA, Kotz KT, Ungaro RF, et al. Microfluidics-based capture of human neutrophils for expression analysis in blood and bronchoalveolar lavage. Lab Invest. 2012;91(12):1787-1795.

16. Nichols JE, Niles JA, Vega SP, et al. Novel in vitro respiratory models to study lung development, physiology, pathology and toxicology. Stem Cell Res Ther. 2013;4(Suppl 1):S7.

17. Kim K, Jung HS, Song JY, et al. Rapid detection of Mycoplasma pneumonia in a microfluidic device using immune agglutination assay and static light scattering. Electrophoresis. 2009;30(18):3206-3211.

18. Shih YF, Huang NT, Lee CK. Capturing CD4 cells using a functionalized circular microfluidic device and glutaraldehyde as bio linker for tuberculosis detection and diagnosis. Microfluidics, Bio MEMS, and Medical Microsystems XIII, San Francisco, California, USA: SPIE library; 2015.
19. Huh D, Fujioka H, Tung YC, et al. Acoustically detectable cellular-level lung injury induced by fluid mechanical stresses in microfluidic airway systems. Proc Natl Acad Sci U S A. 2007;104(48):18886-18891.

20. Huh D, Fujioka H, Grotberg JB, et al. Microfluidic pulmonary system for experimental investigation of cellular injury due to fluid mechanica stresses during airway reopening. In: R. Magjarevic, JH Nagel editors. World Congress on Medical Physics and Biomedical Engineering. Berlin, Heidelberg, Germany: Springer; 2007;14:258-262.

21. Tavana H, Kuo CH, Lee QY, et al. Dynamics of liquid plugs of buffer and surfactant solutions in a micro-engineered pulmonary airway model Langmuir. 2010;26(5):3744-3752.

22. Blume $\mathrm{C}$, Reale $\mathrm{R}$, Held M, et al. Temporal monitoring of differentiated human airway epithelial cells using microfluidics. Plos One. 2015;10(10):e0139872.

23. Skolimowski M, Nielsen MW, Abeille F, et al. Microfluidic model of cystic fibrosis bronchi. $15^{\text {th }}$ International conference on miniaturized systems for chemistry and life sciences; Seattle, Washington, USA: Technical University of Denmark; 2012.

24. Foudeh M, Fatanat Didar T, Veres T, et al. Microfluidic designs and techniques using lab-on-a-chip devices for pathogen detection for pointof-care diagnostics. Lab Chip. 2012;12(18):3249-3266.

25. Tsai TT, Shen SW, Cheng CM, et al. Paper-based tuberculosis diagnostic devices with colorimetric gold nanoparticle. Sci Technol Adv Mater. 2013;14(4):044404.

26. Sackmann EK, Berthier E, Schwantes EA, et al. Characterizing asthma from a drop of blood using neutrophil chemotaxis. Proc Natl Acad Sci U $S$ A. 2014;111(16):5813-5818.

27. Wong, CM Ho. Surface molecular property modifications for poly (dimethylsiloxane) (PDMS) based microfluidic devices. Microfluid Nanofluidics. 2009;7(3):291-306.

28. Halldorsson S, Lucumi E, Sjöberg RG. Advantages and challenges of microfluidic cell culture in polydimethylsiloxane devices. Biosens Bioelectron. 2015;63:218-231. 